This report was prepared as an account of work sponsored by the United States Government. Felther the United States nor the United States Department of Energy, nor any of thete employees, nor any of theif contractors, subcontractors, or their employees, makes any warranicy, express or implied, or assumes any legal liablilty or responsiblity for the accuracy, completeness or ueffulness of any information, apparatus, product or process disclosed, or represepts that its use wold not infeinge privately owned rights.

Printed in the Ondted States of America

$$
\text { Avallable from: }
$$

Mational Technical Information Service

U.S. Department of Comerce

5285 Port Royal Road

Springtield, Virginia 22161

Prtce Prfinted Cogy $\$$ _ , Microfiche $\$ 4.50$

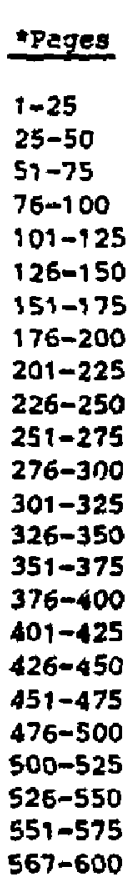

NTIS

Selling Price

$\begin{aligned} \$ 7.00 & \text { For documents over } 600 \\ \$ 8.50 & \text { prges, add } \$ 1.50 \text { for } \\ \$ 10.00 & \text { each additionil 25-page } \\ \$ 11.50 & \text { increment. }\end{aligned}$

$\$ 13,00$

$\$ 14.50$

$\$ 16.00$

$\$ 17.50$

$\$ 19.00$

$\$ 20.50$

$\$ 22.00$

$\$ 23.50$

$\$ 25.00$

$\$ 26.50$

$\$ 28.00$

$\$ 29.50$

$\$ 31.00$

$\$ 32.50$

$\$ 34.00$

$\$ 35.50$

$\$ 37.00$

$\$ 38.50$

$\$ 40.00$

$\$ 41.50$ 
PPPL-2361 (July, 1986)

\title{
Mapping from Rectangular to Harmonic Representation
}

\author{
W: Sclineider* and Glenn Bateman \\ Plasma Physics Laboratory, Princeton University \\ Princeton, New Jersey 08544
}

An algorithm is developed to determine the Fourier harmonics representing the level contours of a scalar function given on a rectangular grid. This method is applied to the problem of computing the flux coordinates and flux surface averages needed for 1-1/2-D transport codes and MHD stability codes from an equilibrium flux function given on a rectangular grid.

\section{DISCLAIMER}

\begin{abstract}
This report was prepared as an account of work sponsored by an agency of the United States Government. Neither the United States Government nor any agency thereof, nor any of their employecs, makes any warranty, express or impliod, or assumes any legal liability or responsibility for the accuracy. completeness, or usefulness of any information, apparatus, product, or procese bisctosed, or represents that its use would not infringe privately owned rights. Reference herein to any specific commercial product, process, or service by trade name, trademark. manufacturer, of otherwise does nol necessably constitute or imply its endorsement, recommendation, or favoring by the United States Government or any agency thereof. The views and opinions of authors expressed herein do nat necessarily state or teflet those of the United States Goveroment of any ageney thereof.
\end{abstract}

-Permanent address: Max-Planck-Institut für Plasmaphysik, EURATOM Association, 8046 Garching bej München, Federal Republic of Germany. 


\section{Introduction}

Given the function $\psi(x, y)$ on a rectangular grid, our objective is to find the inverse functions $x(\psi, \theta)$ and $y(\psi, \theta)$. Ini particular, we wish to deterujine the coefficients $X_{\mathrm{cm}}\left(\psi^{2}\right), X_{\mathrm{sm}}\left(\psi^{4}\right), Y_{\mathrm{mm}}(\psi)$, and $Y_{\mathrm{sm}}\left(\psi^{t}\right)$ in the truncated Fourier series

$$
\begin{aligned}
& \bar{x}\left(\psi^{\prime}, \theta\right)=\sum_{m=0}^{M}\left[X_{m m}(\psi) \cos (m \theta)+X_{m m}\left(\psi^{i}\right) \sin (m \theta) !\right. \\
& \bar{y}(\psi, \theta)=\sum_{m=0}^{M}\left[Y_{c m}(\psi) \cos (m \theta)+Y_{m m}\left(\alpha^{\prime}\right) \sin (m \theta)\right],
\end{aligned}
$$

such that the harmonic representations $\bar{x}(\psi, \theta)$ and $\bar{y}(\psi, \theta)$ most closely approximate the inverse functions $x(\psi, \theta)$ and $y(\psi, \dot{\theta})$. Once the $4 M+2$ Fourier coefficients $X_{\mathrm{cm}}, X_{\mathrm{s}}, Y_{\mathrm{cm}}$, and $Y_{s m}$ (not counting $X_{s 0}$ and $Y_{s 0}$, which have no effect on $\tilde{x}$ and $\tilde{y}$ ) have been determined for $m=0, \ldots, M$, for a sufficiently dense set of $\psi$ values within the range of interest, then $X_{c m}(\psi), X_{a m}\left(\psi^{*}\right), Y_{c m}(\psi)$, and $Y_{s m}(\psi)$ can be determined as needed by interpolation.

\section{Least Squares Fit}

The essential idea of our method is to vary the harmonic coefficients $X_{c m}, X_{s m}, Y_{c m}$, and $Y_{s m}$ until a least squares fit is found for any given level contour $\psi(x, y)=\Psi$. For this purpose, we choose to evaluate $\bar{x}\{\Psi, \theta\rangle$ and $\tilde{y}(\Psi, \theta)$ at a set of at least $4 M+2$ angles $\theta_{i}, i=1, \ldots, I \geq 4 M+2$. (Fewer angles and fewer harmonics are needed if any symmetry conditions are known to prevail.) If this set of angles is held fixed, the functions $\sin \left(m \theta_{i}\right)$ and $\cos \left(m \theta_{i}\right), m=0, \ldots, M$ and $i=1, \ldots, I$ can be evaluated once and stored for computational efficiency. For any given value of $\Psi$, the coefficients $X_{c m}, X_{a m}, Y_{c m}$, and $Y_{s m}$ are varied until a minimum is found for

$$
W=\sum_{i=1}^{I}\left\{\Psi-\psi\left[\tilde{x}\left(\Psi, \theta_{i}\right), \tilde{y}\left(\Psi, \theta_{i}\right)\right]\right\}^{2} .
$$

In Eq. (3), the Fourier series (1) and (2) are evaluated to find $\bar{x}\left(\Psi, \theta_{i}\right)$ and $\tilde{y}\left(\Psi, \theta_{i}\right)$. Then, a two-dimensional interpolation is carried out to find $\psi(\tilde{x}, \bar{y})$ 
from the values given on the rectangular grid. This process is repeated while the harmonic coefficients are varjed until the function $W$ given by Eq. (3) is mininized.

If the interpolation of $\psi(\tilde{x}, \tilde{y})$ is carried out by conventional methods (such as using piecewise polynomials), then the first and second derivatives of $\psi(\tilde{x}, \bar{y})$ can easily be computed along with the interpolated value. In that case, we can explicitly compute smooth approximations to the first and second partial derivatives of $W$ with respect to the harmonic coefficients with very little extra computational work. This makes the minimization of $\mathrm{W}$ much easier and faster.

This method should work particularly well if only a few harmonics are needed to approximate the inverse functions $x\left(\Psi, \theta_{i}\right)$ and $y\left(\Psi, \theta_{i}\right)$ the desired accuracy. The amount of computational effort depends on the number of harmonics needed, not on the number of grid points used in the original rectangular mesh. This method also works best when a good initial guess is already in hand and needs only to be refined by further minimizing $W$ in Eq. (3). The method works well, for example, when the level contours are changing slowly in time so that only a few iterations are needed each time the harmonics need to be updated.

\section{Harmonic Optimization}

One essential problem with the method described above is that the harmonic coefficients needed to minimize $W$ (i.e., to approximate a given closed curve level contour) are not unique. The shape of each level contour is determined by $2 M+1$ constraints on the harmonic coefficients, while the remaining $2 M+1$ constraints needed to determine all $4 M+2$ harmonic coefficients merely determine the disposition of the anglelike variable $\theta$ that parameterizes the curve. In order to see this, suppose $\theta$ is chosen to be the polar angle $\theta_{p}$ around any given point $\left(x_{0}, y_{0}\right)$ jnside a simple closed curve. The shape of the curve can be represented by prescribing the distance $\rho\left(\theta_{p}\right)$ between $\left(x_{0}, y_{0}\right)$ and each point on the curve

$$
\rho\left(\theta_{p}\right)=\sum_{m=0}^{M_{p}}\left[\rho_{c m} \cos \left(m \theta_{p}\right)+\rho_{s m} \sin \left(m \theta_{p}\right)\right] .
$$


This can be converted to rectangular form using

$$
\begin{aligned}
& x=x_{0}+\rho \cos \theta_{p}, \\
& y=y_{0}+\rho \sin \theta_{p},
\end{aligned}
$$

to find the Fourier harmonics in Egs. (1) and (2) for the particular choice $\theta=\theta_{p}$. Equations (1) and (2) result if the number of polar harmonics $M_{p}$ is chosen to be $M-1$. Consequently, the shape of a simple closed curve is determined by $2 . M p+3=2 M+1$ variables

$$
x_{0}, y_{0}, \rho_{c 0}, \ldots, \rho_{c M} t_{p}, \rho_{s 1}, \ldots, \rho_{s, r_{p}}
$$

(not counting $\rho_{\text {so }}$ ) or the corresponding harmonics in Eqs. (1) and (2). Transforming to any other anglelike variable

$$
\theta=\theta_{p}+f\left(\theta_{p}\right)
$$

where $f$ is any periodic function with period $2 \pi$, the lines of constant angle are moved around on the curve, and the Fourier coefficients in Eqs. (1) and (2) change, but the shape of the curve remains unchanged.

Following the work of Hirshman and Mejer, ${ }^{1}$ we establish an algorithm which minimizes the lijgher harmonic coefficjents while leaving the shape unchanged in order to find the most rapidly converging Fourier series that approximates each level contour. This is accomplished by minimizing a penalty function $P$ which increases rapidly with the amplitude of the highest harmonics, using only varjations which leave W [defined by Eq. (3)] unchanged. An example of such a function is

$$
P=\frac{\sum_{m=1}^{M} m^{p+q}\left(X_{c m}^{2}+X_{s m}^{2}+Y_{c m}^{2}+Y_{s m}^{2}\right)}{\sum_{m=1}^{M} m^{p}\left(X_{c m}^{2}+X_{s m}^{2}+Y_{c m}^{2}+Y_{s m}^{2}\right)},
$$

where $p$ and $q$ are positive exponents. This function reaches its absolute minimum value $P=1$ when all but the first harnonics are zero. If all the harmonics are rescaled, $P$ remains unchanged. The zeroth harmonics $X_{c 0}$ and $Y_{c 0}$ have no effect on $P$, since they influence only the position and not the shape of the curve. There are many suitable functions with these properties; the particular choice made in Eq. (4) is not important. 
The function $P$ is to be mininized subject to the constraint that $W$ renains unchanged. Suppose the array $\mathrm{C}=\left(C_{1}, \ldots, C_{4 M+2}\right)$ represents the set of all the harmonics being considered

$$
C=\left(X_{c 0}, \ldots, X_{c M}, X_{21}, \ldots, X_{s M}, Y_{c 0}, \ldots, Y_{c M}, Y_{91}, \ldots, Y_{s M}\right) \text {. }
$$

Variations of $\mathbf{C}$ which leave $\mathbb{W}$ unchanged (and, therefore, variations which run parallel to the level contour being sought) are given by

$$
\delta C_{\| m}=\delta C_{m}-\frac{\sum_{i} \frac{\partial W^{r}}{\partial C_{1}} \delta C_{2}}{\sum_{j}\left(\frac{\partial W}{\partial C_{j}}\right)^{2}} \frac{\partial W}{\partial C_{m}} .
$$

The variation of $P$ subject to this constraint is then given by

$$
\delta P_{\|}=\sum_{m=1}^{4 M+2} \frac{\partial P}{\partial C_{m}} \delta C_{\| m}
$$

Lising Eq. (6) and rearranging, we gei

$$
\delta P_{\|}=\sum_{m}\left[\frac{\partial P}{\partial C_{m}}-\frac{\sum_{i} \frac{\partial W}{\partial C_{1}} \frac{\partial P}{\partial C_{i}}}{\sum_{j}\left(\frac{\partial W}{\partial C_{j}}\right)^{2}} \frac{\partial P}{\partial C_{m}}\right] \delta C_{m} .
$$

A constrained minimum, ihen, is achieved when $\delta P_{\|}=0$ with arbitiary variations $\delta C_{m}$. This results in the system of equations

$$
\frac{\partial P}{\partial C_{m}}-\frac{\sum_{i} \frac{\partial W}{\partial C_{i}} \frac{\partial P}{\partial C_{i}}}{\sum_{j}\left(\frac{\partial W}{\partial C_{3}}\right)^{2}} \frac{\partial W}{\partial C_{m}}=0 \quad \text { for } \quad m=1, \ldots, 4 M+2 .
$$

These equations need to be solved together with the equations needed for the unconstrained minimization of $W$

$$
\frac{\partial W}{\partial C_{m}}=0 \quad \text { for } \quad m=1, \ldots, 4 M+2 .
$$

Equations (9) and (10) taken together represent $8 M+4$ equations for the $4 M+2$ variables in array $C$. In spite of this, however, we will now show that this combined system of equations is not overdetermined. Let

$$
W_{m} \equiv \frac{\partial W}{\partial C_{m}}, \quad P_{m} \equiv \frac{\partial P}{\partial C_{m}}
$$


and

$$
w_{m} \equiv \frac{W_{m}}{\sqrt{\sum_{j=1}^{4 \sqrt{l+2} W_{j}^{2}}}} .
$$

Since the values of $W_{\mathrm{m}}$ may converge to zero at different rates as we converge to a solution of $\mathrm{Eq}$. (I0), the values of $\omega_{\mathrm{m}}$ are left free (and, in general, nonzero). The constraint equations (9), which may be written

$$
P_{m}=\sum_{i=1}^{4 M_{+}^{+2}} u_{i} P_{i} w_{m}
$$

imply

$$
w_{m}=\frac{P_{m}}{\sqrt{\sum_{i} P_{i}^{2}}} .
$$

As long as the values of $W_{m}$ converge to zero independent of one another, the values of $w_{m}$ (and, consequently $P_{m}$ ) are free, und Eq. (9) imposes no constraint on $P_{m}$.

Suppose, however, there are $K$ linearly dependent relations among the values of $W_{m}$ as they converged to zero

$$
\sum_{j=1}^{4 M+2} a_{j k} W_{j}=0, \quad \text { for } \quad k=1, \ldots, K .
$$

It then follows that

$$
\sum_{j=1}^{4 M+2} a_{j k} w_{j}=0, \quad \text { for } \quad k=1, \ldots, K
$$

and, from Eq. (14),

$$
\sum_{j=1}^{4 M+2} a_{j k} P_{j}=0, \quad \text { for } \quad k=1, \ldots, K .
$$

Hence, any linear dependence among the values of $W_{m}$, as they converge to zero, results in a corresponding linear dependence constraint on the values of $P_{m}$. Under these conditions, there would be $4 M+?-K$-onst raints from Eqs. (10) and $k$ constraints from Eqs. (9). Conseouently, there are a total of $4 M+2$ constraining equations for the $4 M+2$ unknowns. 


\section{Singular Value Decomposition}

The well-known technique of singular value decomposition ${ }^{2,3}$ can be used to isolate any linear dependence among the minimizing Eqs. (10). In order to see how this is done in the present context, Taylor series expand Eq. (10)

$$
\frac{\partial W(\mathbf{C})}{\partial C_{m}}=\frac{\partial W\left(\mathbf{C}^{1}\right)}{\partial C_{m}}+\sum_{n} \frac{\partial^{2} W\left(\mathbf{C}^{1}\right)}{\partial C_{m} \partial C_{n}}\left(\mathbf{C}-\mathbf{C}^{1}\right)_{n}+\cdots
$$

where $\mathbf{C}^{1}$ is the value of $\mathbf{C}$ at the current iteration. Neglect higher order terms and do a singular value decomposition of the Hessias matrix

$$
\frac{\partial^{2} W}{\partial C_{m} \partial C_{n}}=\sum_{j} U_{m j} \sigma_{j} V_{j n}
$$

where $U$ and $V$ are orthogonal matrices (i.e., $U^{-1}=U^{t}$ ) and

$$
\sigma_{j}, \quad j=1, \ldots, 4 M+2
$$

are the singular values. Multiply Eq. (17) on the left by $U^{-1}$ and look for conditions under which $\mathbf{C}$ is a solution to

$$
\frac{\partial W(\mathrm{C})}{\partial C_{m}}=0
$$

to obtain

$$
\sum_{m} U_{j m+}\left(\mathbf{C}^{1}\right) \frac{\partial W\left(\mathbf{C}^{1}\right)}{\partial C_{m}}=\sigma_{j} \sum_{n} V_{j n}\left(\mathbf{C}-\mathbf{C}^{3}\right)_{n}
$$

If all the singular values $\sigma_{j}$ are nonzero, then none of the equations for $\partial W / \partial C_{m}$ are linearly dependent, and there are no furthor constraints imposed by $\mathrm{Eq}$. (9). However, if any of the singular values are zero, $\sigma_{j}=0$, then

$$
\sum_{m} U_{j m}\left(\mathbf{C}^{1}\right) \frac{\partial W\left(\mathbf{C}^{1}\right)}{\partial C_{m}}=0,
$$

over that range of values of $\mathbf{C}^{\mathbf{l}}$ in the neighborhood of the minimum of $W$ where we neglected higher order terms in the Taylor series (17). For each of these linear relatious among the equations for $W_{m}$, it follows from $\mathrm{Eq}$. (16) that there is the same linear relation among the values for $P_{m}$

$$
\sum_{m} U_{j m}\left(\mathbf{C}^{1}\right) \frac{\partial P\left(\mathbf{C}^{1}\right)}{\partial C_{m}}=0
$$


Hence, we have the following $4 M+2$ equations

$$
\begin{array}{ll}
\sum_{m} U_{j m}(\mathbf{C}) \frac{\partial W(\mathbf{C})}{\partial C_{m}}=0 & \forall_{j \text { st } \sigma_{j} \neq 0}, \\
\sum_{m} U_{j m}(\mathbf{C}) \frac{\partial P(\mathbf{C})}{\partial C_{m}}=0 & \forall_{j \text { st } \sigma_{j}=0}
\end{array}
$$

to deterssine the $4 M+2$ elements of the array $C$.

\section{Implementation}

It is useful to describe the way in which the above method was inplemented, in order 1.0 point out how various problems with the implenentation were solved.

First, the two-dimensional array of $\psi(x, y)$ values is searched to find the minimum or maximum values of interest. These minimax grid points are tested to deternine which are elliptical (true minima or maxima, re. ferred to as o-points), and which are hyperbolic (sacldle points, referred to as $x$-points). One elliptical point is chosen and the true position $\left(x_{0}, y_{0}\right)$ of that minimum or maximum is found, consistent with the interpolation being used. A suitable range is found for $\psi$ which must not. include any other minimax points. Particular care must be taken to avoid hyperbolic points, since the harmonic representations (1) and (2) will not converge on contours passing through these x-points (separatrices). A set of $\psi^{r}$ values is chosen in this range on which the coefficients of the inverse functions will be determined, working from the center to the edge.

Niear the c-point, the contours are nearly elliptical, and the inverse functions can be initially approximated by

$$
\begin{aligned}
& x \approx \alpha_{0}+x_{1}, \sin \theta+x_{1 c} \cos \theta, \\
& y \approx y_{0}+y_{1} \sin \theta+y_{1 c} \cos \theta
\end{aligned}
$$

Using this representation, we can choose $y_{1 c}=0$ and choose positive values for the other coefficients in order to place the point where $\theta=0$ on the horizontal line $y=y_{0}$ to the right of the minimax point. The coefficients can then be determined analytically from the polynomial approximation to 
$L^{\prime}(x, y)$ near the o-point. These coefficients can be used as the first iterate for the inverse functions representing the contour closest to the o-point.

As long as the function $\psi(x, y)$ is essentially parabolic near the o-point, the harmonic coefficients scale like $h^{m / 2}$ where

$$
h \equiv\left|\frac{4 \cdot-\psi_{\min }}{\xi_{\max }-\xi_{\min }}\right| .
$$

The scaled coefficients are extrapclated away from the o-point in order to provide initial iterates for each successive contour, working out to the edge. If a good initial iterate is already available from previous solutions for the harmonics, then a weighted average is taken of the two.

Probles.ns most frequently occur near the outermost contour. If this contour is located near a separatrix, the higher harmonic amplit udes increase dramatically, making the initial extrapolation and subsequent solution more difficult. Since the $x$-point on the separatrix contour is a saddle point, the $\left[\bar{x}\left(\Psi, \hat{\theta}_{i}\right), \bar{y}\left(\Psi, \theta_{i}\right)\right]$ points being evaluated in Eq. (3) can inad vertently get. caught on the wrong side of the saddle, with no gradient to drive it back toward the region of interest. Near such a saddle point, and also near the edge of the rectangular domain, more care needs to be taken in the choice of interpolation used to eve luate $\psi(\tilde{x}, \tilde{y})$. For these reasons, cautious nethods of extrapolation and nonlinear equation solving were used, particularly for the outer contours.

\section{Jacobian and Metric Elements}

Once the inverse functions ( 1 ) and (2) have been determined for a given choice of parameters $\psi$ and $\theta$, it is a straightforward matter to compute the Jacobian

$$
\mathcal{J}(\psi, \theta)=\frac{\partial x}{\partial \psi} \frac{\partial y}{\partial \theta}-\frac{\partial x}{\partial \theta} \frac{\partial y}{\partial \psi},
$$

metric elements

$$
\begin{aligned}
& g_{\psi \psi \psi}=\left(\frac{\partial x}{\partial \psi}\right)^{2}+\left(\frac{\partial y}{\partial \psi}\right)^{2}, \\
& g_{\theta \theta}=\left(\frac{\partial x}{\partial \theta}\right)^{2}+\left(\frac{\partial y}{\partial \theta}\right)^{2},
\end{aligned}
$$




$$
g_{\psi \theta}=g_{\theta \downarrow}=\frac{\partial x}{\partial \psi} \frac{\partial x}{\partial \theta}+\frac{\partial y}{\partial \psi} \frac{\partial y}{\partial \theta}
$$

area,

$$
A=\int^{\downarrow} d \psi \cdot \int_{0}^{2 \pi} d \theta \cdot T(\psi, \theta)
$$

and contour integral,

$$
\oint d l f=\int_{0}^{2 \pi} d \theta \sqrt{\left(\frac{\partial c}{\partial \theta}\right)^{2}+\left(\frac{\partial y}{\partial \theta}\right)^{2}} f\left(\psi^{\prime}, \theta j .\right.
$$

The Fourier representaion maties it particularl/ easy to take $\theta$ derivatives. We use cubic spline interpolation ${ }^{4,5}$ in $\psi$ t the $\psi$ derivatives and Fast Fisurier Transform ${ }^{4,5}$ techniques to take $\mathrm{p}$. is and evaluate the other nonlinear finctions.

For applications involving axisymmetric figures of rotation, such as tokamak equilibria, we wish to map from a polar coordinate system centered on the axis of rutation $(R, Y, \phi)$ to a "flux coordinate system" $(\psi, \theta, \phi)$ given the flux function $\psi(R, Y)$. Then, the Jacobian is

$$
\mathcal{J}(\psi, \theta, \phi)=R\left(\frac{\partial R}{\partial \psi} \frac{\partial Y}{\partial \theta}-\frac{\partial R}{\partial \theta} \frac{\partial Y}{\partial \psi}\right),
$$

and the volume is

$$
V=2 \pi \int^{\psi} d \psi \int_{0}^{2 \pi} d \theta \mathcal{J}(\psi, \theta, \phi)
$$

\section{Applications}

Two examples are shown in Figs, 1 and 2 of axisymmetric MHD equilibria that were computed in rectangular $(R, Y, \phi)$ coordinates and then mapped to flux coordinates using the procedure described in this report. In each case, four harmonics were used to represent the inverse functions $R(\psi, \theta)$ and $Y(\psi, \theta)$ on each of 20 level contours. It was found that using fewer than four harmonics resulted in an observable discrepancy in the shape of the outer flux surface at the tip of the bean in Fig. 1, while using more than four harmonics led to no significant further change in the 
platted results or the computed flux surface averages (Jacobian or metric elements). The exponents $p=1$ and $q=4$ were used in Eq. (4), which resulted in rapidly converging harmonic representations.

Both of the computed flux functions $\psi(R, Y)$ for these equilibria have reflection symmetry across the midplane. This syzametry condition was used to reduce the number of harnonic coefficients needed in Eqs. (1) and (2) and also to reduce the number of angles needed to evaluate Eq. (3). For the examples presented here, 12 equally spaced angles $\theta_{i}$ were used in the upper half plane. The number of angles needed depends partly on the number of harmonics used (we need at least as many angles as harmonic coefficients) and partly on the spacing needed to resolve details of the level contour shape, particularly near the x-point of a se paratrix. It is important to keep in mind that the anglelike variable $\theta$ is generally not the polar angle. The harmonic optimization procedure [Eq. (4)] helps to resolve details of the level contours with the fewest harmonics and also with the fewest angles $\theta_{i}$ in Eq. (3). Essentially, the optimization procedure tends to force equally spaced angles $\theta_{i}$ to dccumulate in regions where the level contour curvature is the greatest, as can be seen in both figures.

During theitteration, linearized forms of Egs. (20) and (21) were solved using $\mathrm{NAG}^{5}$ routines F02WCF to find the singular value decomposition and F02ARF to solve the linear equations for $C$. On the first pass through, the PBX equilibrium shown in Fig. 1 typically needed five to six iterations per flux surface while the ASDEX case shown in Fig. 2 required only three iterations for each of the inner 17 flux surfaces and up to six iterations for the outer three surfaces. On a CRAY-1 computer, this entire mapping procedure took $2.0 \mathrm{cpu}$ seconds for the PBX case and $1.15 \mathrm{cpu}$ seconds for the ASDEX case.

Clearly, the most difficult part of the computation is near the outer contours. Relative to the interval of $\psi$ between the innermost contour and th $>$ separatrix [saddle point in $\psi(R, Y)$ ], the outer contour shown in Fig. 1 is $97 \%$ of the way to the separatrix. In this respect, the ASDEX case was a more difficult configuration; the outer contour shown in Fig. 2 is only 93\% of the way to the separatrix.

Since the function $\psi(R, Y)$ is the solution of an elliptic equation, and consequently very smooth, for the cases presented here, sixth order poly- 
nomials in both $R$ and $Y$ were used to interpolate $\psi(R, Y)$ on the evenly spaced $(R, Y)$ grid to determine the inner contours, while lower order interpolation was used near the edge. This was found to be more accurate and to use less computer memory than bicubic splines. The plasmas shown in these examples do not cover the entire rectangular grid, since ofher structures (liniters, walls, divertors and coils, not shown) had to also fit on the grid in the origiral equilibrium computations. While the entire rectangular grid in the PBX case (Fig. 1) contains 51 horizontal by 61 vertical poiais, there are only 14 grid points across the midplane of the plasma and 40 points from the top to bottom tips of the plasma. In the ASDEX case (Fig. 2), a 61 by $\$ 01$ grid was used with 32 by 55 points between the extremities of the plasma. One of the advantages of the method presented in this paper compared to most conventional contour tracing routines is that the contours remain smooth even when the rectangular grid is coarse.

The examples described above demonstrate the capability of the method under the realistic conditions for which it was developed, but they do not provide known absolute measures with which $t s$ compare the accuracy of the results. For this purpose we computed the area enclosed by elliptical flux surfaces prescribed analytically. The results depend on the coarseness of the rectangular grid, on the method of interpolating on this grid, on the number of angles $\theta_{i}$ used in $\mathrm{Eq}$. (3), and on the method used to integrate Eq. (26). Using a 32 by 32 grid, fourth order local interpolation, 20 angles $\theta_{i}$, and cubic spline integration of Eq. (26) over 20 surfaces, the area was computed with relative error $4 \times 10^{-7}$.

\section{Conclusions}

The method normally used to determine and integrate along level contours involves tracing out each contour by computing the local intersections of the contour as it crosses each line of the rectangular grid. Compared with this standard method, our mapping procedure has the following advantages: The computed harmonic representation is relatively insensitive to the coarseness of the rectangular grid. The mapping produces a compact parametric represenfation of a curvalinear coordinate system, from which the Jacobian and metric elements can be computed. The representation 
uses an optimal choice for the parameter $\theta$ to make the Fouriet series (1) and (2) converge as rapidly as possible. Hence, this representation provides a good starting point for mapping to any other curvalinear coordinate system that follows the level contours (fux coordinates). ${ }^{6}$ The computational time depends on the number of harmoriscs needed and the number of angles $\theta_{i}$ used to evaluate Eq. (3), not on the number of rectangular grid points. The iteration benefits from any previous computations, provided the contour shapes have not changed very much.

Mapping to harmonic representation in the form presented here cannot be used in multiply connected regions or in the neighborhood of a separatrix. The method must be limited to a region of simply nested level contours. When approaching a separatrix, the user must be cautious to avoid reaching out beyond the separatrix during the iteration.

When applying this method to a new problem, the user must verify that. enough harmonics and evaluation angles $\theta_{i}$ in Eq. (3) are used. There is some incentive to chonsing as few harmonics and angles as needed in order to minimize computational time. Compared to contour tracing, there is generally less interpolation on the rectangular grid, but some vhat more effort needed to compute the singular value decomposition and the solution of Eqs. (20) and (21).

\section{Acknowledgments}

We wish to thank Dr. S. C. Jardin for the use of his TCS code to compute the equilibria shown in figs. 1 and 2.

This work was supported by U.S. DOE Contract No. DE-AC02-76CHO-3073. 


\section{References}

${ }^{1}$ S. P. Hirshman and H. K. Meier. Phys. Fluids 28 (1985) 1387.

${ }^{2}$ George E. Forsythe, Michael A. Malcolm, and Cleve B. Moler. Computer Methads for Mathematical Computations. Prentice-Hall, Englewood Cliffs, NJ, 1977.

${ }^{3}$ Charles L. Lawson and Richard J. Hanson. Solving Least Squares Problems. Prentice-Hall, Inc., Englewood Cliffs, NJ, 1974.

${ }^{4}$ IMSL Library User's Manual: FORTRAN Subroutines for Mathematics and Statistics. IMSL, NBC Building, 7500 Bellaire Boulevard, Houston, Texas 77036-5085, November 1984.

${ }^{5}$ NAG FORTRAN Libnary Manual. Numerical Algorithms Group, 1250 Grace Court, Downer's Grove, Illinois 60516, 1982.

${ }^{6}$ Glenn Bateman. MHD Instabilities. MIT Press, Cambridge, Mass., 1978. 


\section{Figures}

FIG. 1. Cross section of the flux coordinates computed for the Princeton Beta Experiment (PBX).

FIG. 2. Cross section of the flux coordinater computed for the ASDFX upgrade tokamak. 


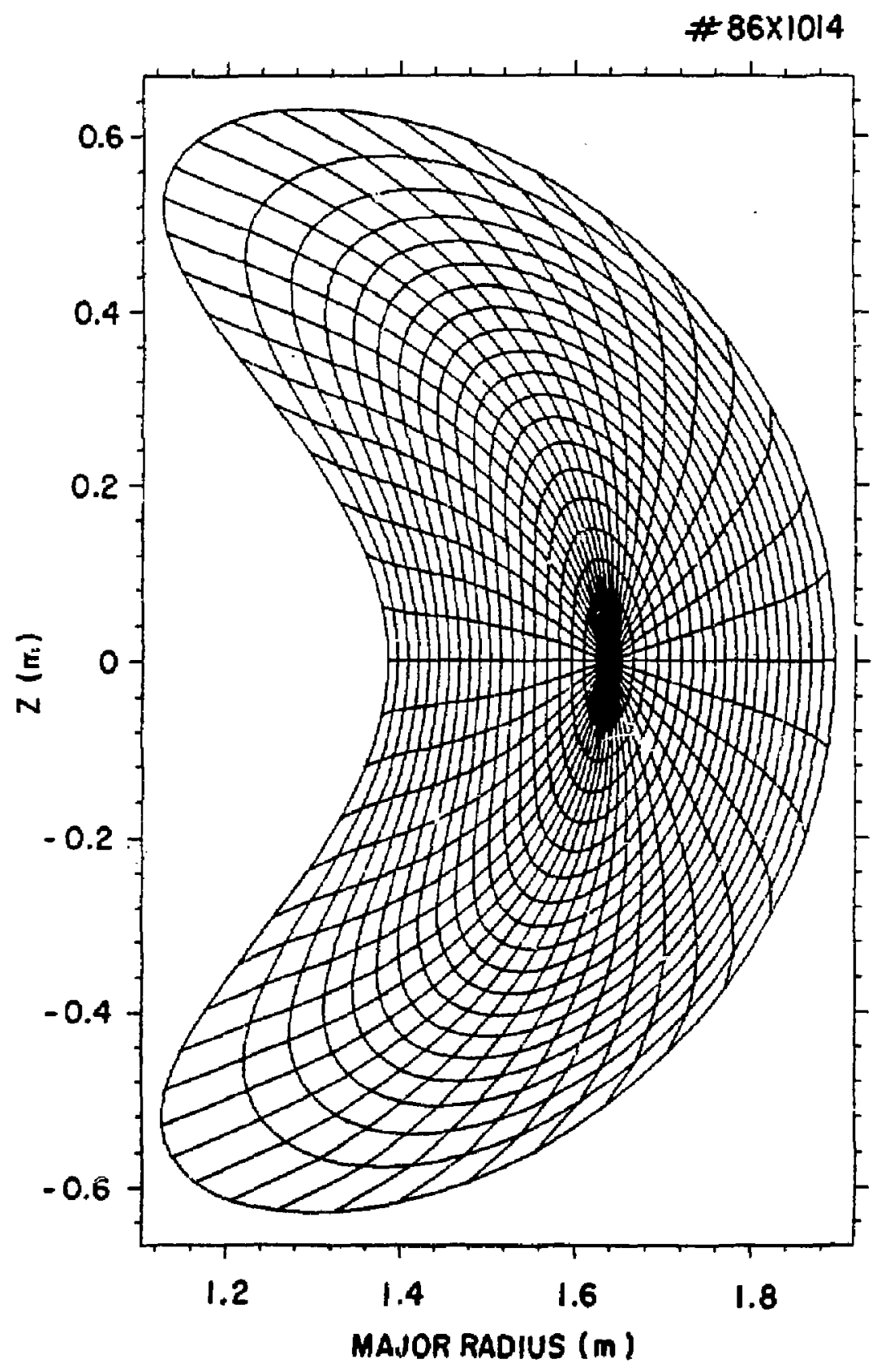

FIG. 1. Crass section of the flux coordinates computed for the Princeton Beta Experiment (PBX). 


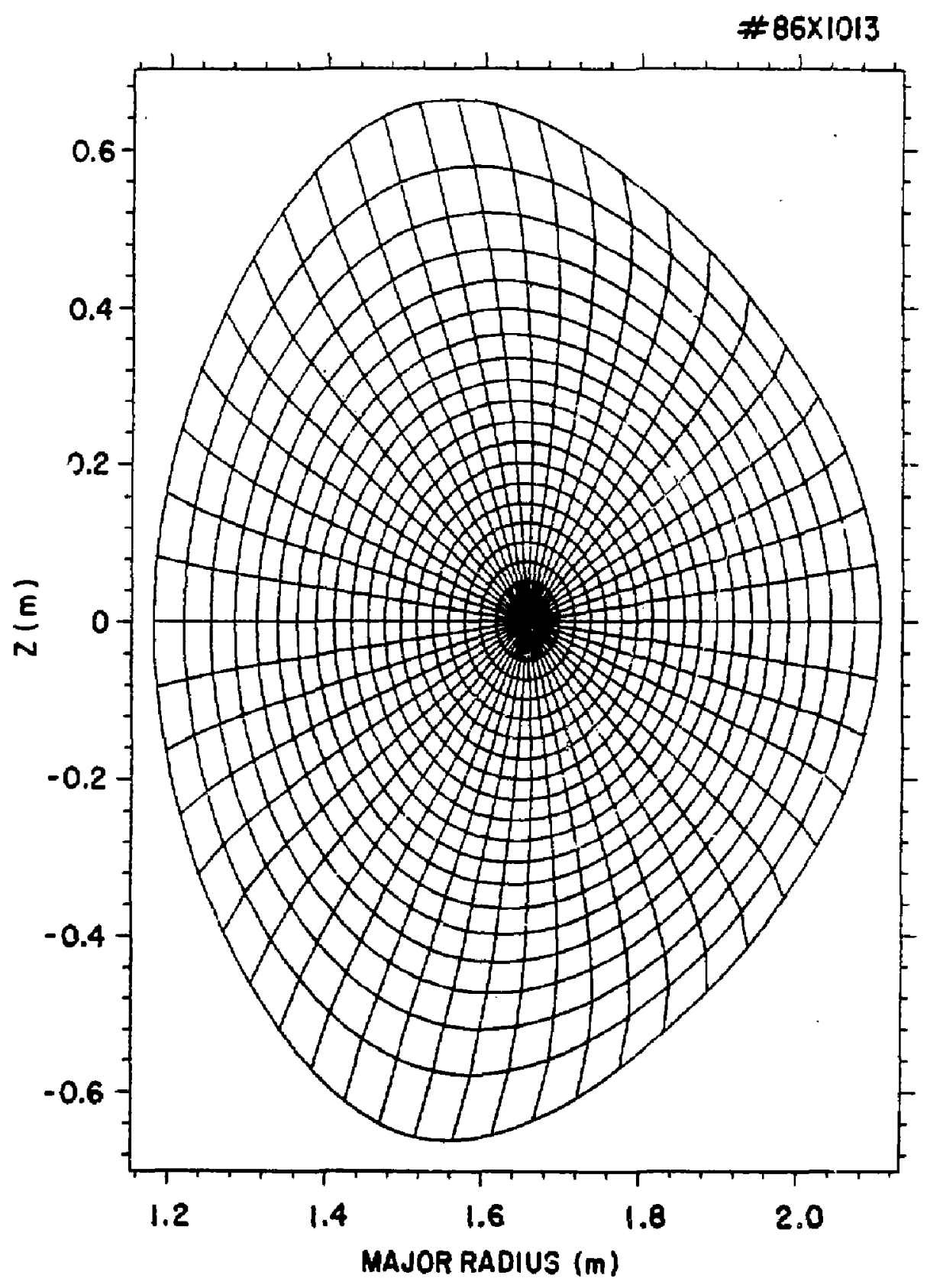

FJG. 2. Cross section of the flux coordinates computed for ASDEX upgrade tokamak. 
Pi-sma Ras Lab, Austra Nat'I UnIv, Australia

Jr. Frank t. Paolonl, Unlv of wol longong. AUSTRAL IA

Prof. I,R. Jones, FIInders Un!W., AUSTRALIA

Prof. M.H. Erennan, Univ Syoney, AuSTRALIA

Prot. F. Gad, Inst Theo Phys, RUSTRIA

H. Goossens, Astronomlseh Instltuut, BELGILM

Prof. R. Bouclaue, Laboratorium voor Matuurkunde, BELGIJM

Dr. D. Palumbo, Dg XI I Fuslon Prog, belgium

Ecole Rayale MIlitalre, Leb de Phys Plasmas, BELGILM

Dr. P.H. Sakanaka, Univ Estadual, BRNZIL

LIb. Doc. Olu., Instituto de Pasquises Espaclala, gRN1L

Or. C.R. Jomes, Univ of Alberta, CANADA

Piof. J. Telchmann, Unlv of Montreal, CANADA

Or. H.M. Skarsgard, Unlv of Saskatehewan, CANADA

Prot. S.R. Sreanivasan, Unlveislty of Celgary, CANAOA

Prot. Tudor W. Johnston, IRRS-Energle, CANADA

Dr. Hanncs Barnard, Univ British Calumbla, Canada

Dr. M.P. Bachynskl, MPB Technologies, Inc, CANAOA

Chalk River, Nucl Lab, CANADA

$Z$ hengwu LI, Sw inst Physles, CHINA

Library, Tsing Hua UnIvorsity, CHINA

LIbrarlan, Institute of Physles. CHINA

Inst Plasme Phys, Academla Sirilco, CHINA

or. Peter Lukac, Kemínskahio UnIy, CECHOSLOVAKIA

The Librartan, Culhan Laboratory, ENGLAND

Prof. Schatzman, Observatel re de NI ce, FRARice

J. Radet, CEN-AP5, FRANCE

JET Reeding Room, JET Joint Undertakirig, ENGLAND

AM Dupas LIbrary, AM Dupes LIbrary, FRANCE

3r. Tom MLAI, Acadeny Blbllographlc, HOHG KONG

Preprint Library, Cant Res Inst Phys, HUNGARY

Or. R,K, Chhojlanl, VIkram Unlu, INolA

Dr. B. Dasgupta, Selta Inst, INOIA

Or. P. Kow, Physical Resezrch Lab, InDIA

Dr. Phlllip Rosenau, Israat Inst Tech, ISRAEL

Prot. S. Cuporman, Tel AvIv University, ISRAEL

Prot. G. Rostagni, Univ DI Padovo, ITALY

LIbrarjan, Int'I Cts Theo Phys, ITAl.Y

Miss cielis De Palo, Assoc ElPATOM-ENEA, ITALY

BIbllotecs, dal CNR ELRATOA, ITALY

Dr. H* Yansto, Toshl ba Res a Doy, JAPAN

Diruc. Dopt. Lg. Tokamak Duv. JAERI, JAPAH

Prot. Nobuyukl Inoue, UnIversity of Tokye, JAPAN

Research info Canter, Nogora University, JAPAN

Prot. Kyojt Nishlkewa, UnIv of HIroshima, JAPAN

Prof. SIgeru Morl, JAERI, JAPAN

Prot. S. Tanaka, Kyoto Unlvarsity, JAPAN

LIbrany, Kyoto UnIversIty, JAPAN

Prof. Ichlro Kawakaml, Nihon Univ, JAPAN

Prot. Satoshl 1toh, Kyushu Unlvarsity, JAPAH

Dr. D.l. Chol, Adv, Inst Sel \& Tech, KOREA

Tech Into OIvIsIOn, KAERI, KOREA
Bibllotheek, Fom-Inst Voor PIasma, NETHERLANDS

Prof. B.S. LIleY, University of Walkato, NEW ZEALANO

Prof. J.A.C. Cabral, Inst Superlor Tecn, PDRTJGaL

Or. Octavlan Petrus, ALI CLEA UnIversity, ROMANIA

Prof. M.A. Hellberg, University of Natal, SO AFRICA

Dr. Johan do Villlers, Plasma Physics. Nucor, SO AFRICA

Fuslan Div. Library, JEN, SPAIN

Prof. Hans Wilhalmson, Cholmers Untr Tecn, SWEDEN

Or. Lennart StentJo, Unlversity of UMEA, SWEDEN

Library, Royal Inst Tech, SWEOEN

Centro de Recherchesen, Ecale Polytech Fad, SwITZERLAND

Dr. V.T. Tolok, Kharkov Phys Tech Ins, USSR

Dr. D.D. Ryutov, SI berlan Acad Sel, USSR

Dr. G.A. Ellseav, Kurchatov Institute, USSR

Dr. V,A. Glukhlkh, Inst Electro+Physlcal, USSR

Institute Gen. Physles, USSR

Prof. F.J.M. Boyd, Un Iv College $\mathrm{N}$ Wales, WALES

Or. K. Schindler, Ruhr Univarsitat, W. GERWANY

ASOEX Readling Rm, IPPMax- lanck-I nstitut fur

Plasmaphyslk, F.R.G.

Nuclaar Res Estab, Julich Lto, W. GERMANY

LIbrarian, Max-Planck Institut, W. GERMANY

Blbllothek, Inst Plasmatorschung, W. GERMANY

Prof, R,X. Janev, I nst Phys, YueOSLAVIA 